\title{
Quarkonia photo-production and Z production in heavy-ion collisions at LHCb
}

\author{
Giulia Manca $^{a, *}$ (on behalf of the LHCb collaboration) \\ ${ }^{a}$ Università degli studi di Cagliari and Istituto Nazionale di Fisica Nucleare, \\ Cittadella Universitaria di Monserrato, 09042 Monserrato (CA),Italy \\ E-mail: giulia.manca@cern.ch
}

The measurements of $\mathrm{Z}$ boson and $\mathrm{J} / \psi$ production in proton-lead and ultra-peripheral lead-lead collisions are presented using data collected by the LHCb detector in 2015 and 2016. The results are compared with several theoretical predictions.

40th International Conference on High Energy physics - ICHEP2020

July 28 - August 6, 2020

Prague, Czech Republic (virtual meeting)

\footnotetext{
* Speaker

The contact author acknowledges support from the European Research Council(ERC) through the project EXPLORINGMATTER, founded by the ERC through a ERC-Consolidator-Grant, GA 647390.
} 


\section{Introduction}

In the last years the LHCb experiment has provided novel inputs to heavy-ion physics by exploiting some of its unique features through the studies of $p p, p \mathrm{~Pb}, \mathrm{PbPb}$ and fixed target collisions at LHC energies for pseudorapidity between 2 and 5 . These measurements cover a kinematic range complementary to the ones of the other major LHC experiments, and reach higher precisions thanks to the excellent vertexing and particle identification capabilities of $\mathrm{LHCb}$, allowing exclusive measurements of open and hidden charm states, including baryons, as well as studies of electroweak gauge bosons in the forward direction. Production measurements in this unique kinematic range provide crucial constraints on models of key interest for parton distribution function and electroweak physics among others. In these proceedings selected results in production of $\mathrm{Z}$ boson in $p \mathrm{~Pb}$ collisions and $\mathrm{J} / \psi$ in ultra-peripheral $\mathrm{PbPb}$ collisions are presented and compared with the available theoretical models.

\section{Detector and simulation samples}

The $\mathrm{LHCb}$ detector $[1,2]$ is a fully instrumented single-arm spectrometer in the forward region covering a pseudorapidity acceptance of $2<\eta<5$, providing a high tracking momentum resolution down to very low transverse momentum $\left(p_{\mathrm{T}}\right)$ and precise vertex reconstruction capability. Table 1 summarises the heavy-ion samples collected by LHCb in Run 2. Simulation is used in the determination of efficiencies. The $p$ - $\mathrm{Pb}$ and $\mathrm{Pb}-\mathrm{Pb}$ collisions are simulated with EPOS-LHC [3] and STARlight [4] and the dimuon decays with РүтніA 8.1 [5] in $p p$ collisions where the proton beam energy is equal to the nucleon beam energy in $p \mathrm{~Pb}$ collisions. The interaction of the generated particles with the detector and its response are implemented using the Geant4 toolkit [6].

\section{3. $\mathbf{Z}$ boson production in $p \mathbf{P b}$ collisions}

The electroweak bosons decaying leptonically are excellent probes since they decay very early and the leptons produced in the decay traverse the medium unmodified. For this reason any difference of their rates in $p p$ vs. $p \mathrm{~Pb}$ and $\mathrm{PbPb}$ collisions can be a clear quantification of effects due to cold nuclear ordinary matter (CNM) and not hot nuclear matter, i.e. Quark Gluon Plasma (QGP). In $\mathrm{LHCb}, \mathrm{Z}$ and W's are used mainly to constrain the nuclear parton distribution functions (nPDFs, which take into account the effect of the several partons in the nucleus), in a region of Bjorken- $x$ $10^{-4}-1[7,8]$, unaccessible by other experiments. In addition, they are valuable as calibrations for studying modification factors in other processes. In this analysis [9] the cross section times

\begin{tabular}{|c|c|c|c|c|c|c|c|}
\hline year & \multicolumn{2}{|c|}{2013} & \multicolumn{2}{|c|}{2016} & 2015 & 2018 & 2010-18 \\
\hline$\sqrt{s_{\mathrm{NN}}}$ & \multicolumn{2}{|c|}{$5.02 \mathrm{TeV}$} & \multicolumn{2}{|c|}{$8.16 \mathrm{TeV}$} & & $\mathrm{TeV}$ & $13 \mathrm{TeV}$ \\
\hline & $p \mathrm{~Pb}$ & $\mathrm{~Pb} p$ & $p \mathrm{~Pb}$ & $\mathrm{~Pb} p$ & & & $p p$ \\
\hline $\mathcal{L}$ & $1.1 \mathrm{nb}^{-1}$ & $0.5 \mathrm{nb}^{-1}$ & $13.6 \mathrm{nb}^{-1}$ & $20.8 \mathrm{nb}^{-1}$ & $10 \mu b^{-1}$ & $-210 \mu b^{-1}$ & $9 \mathrm{fb}^{-1}$ \\
\hline
\end{tabular}

Table 1: Data samples collected by the LHCb experiments in Run 2. 
branching ratio is measured in $p \mathrm{~Pb}$ collisions in two rapidity ranges, integrated over $p_{\mathrm{T}}$, according to the formula $\sigma_{Z \rightarrow \mu^{+} \mu^{-}}=\frac{N_{Z} \cdot \rho}{\mathcal{L} \cdot \epsilon_{T O T}}$, where $N_{Z}$ is the number of signal events, $\rho$ is the purity of the sample, $\mathcal{L}$ is the luminosity and $\epsilon_{T O T}$ is the total efficiency. The $p \mathrm{~Pb}$ collision data were divided in two different configurations, the forward $(p \mathrm{~Pb})$, where the proton beam travels from the VELO detector to the muon chambers, and the backward $(\mathrm{Pb} p)$, where the proton and lead beams are inverted $^{1}$. The energy of the proton beam is $6.5 \mathrm{TeV}$, while that of the lead beam is $2.56 \mathrm{TeV}$ per nucleon, resulting in a centre-of-mass energy of the proton-nucleon system of $8.16 \mathrm{TeV}$. Since the energy per nucleon in the proton beam is significantly larger than that in the lead beam, the proton-nucleon centre-of-mass system has a rapidity in the laboratory frame of $+0.465(-0.465)$ for $p \mathrm{~Pb}(\mathrm{~Pb} p)$ collisions, resulting in a shift of the range of the centre-of-mass rapidity $y^{*}$ in the proton nucleus case. This results in a coverage of $1.5<y^{*}<4.0$ for $p \mathrm{~Pb}$ forward and $-5.0<y^{*}<-2.5$ for $\mathrm{Pb} p$ backward collisions. The $\mathrm{Z}$ bosons are reconstructed though their decay into two oppositesign muons of $p_{\mathrm{T}}>20 \mathrm{GeV} / c$, rapidity $2.0<\eta<4.5$, and forming an invariant mass in the window $60-120 \mathrm{GeV} / c^{2}$ around the $\mathrm{Z}$ nominal mass of $91 \mathrm{GeV} / c^{2}$. The total number of signal events is 268(167) in the forward(backward) sample, as shown in Fig. 1, left, where the data are compared with Pythia 8.1 [5] with CTEQ6L1 [10] PDFs set, normalised to the number of observed candidates. $\rho$ is estimated using the number of same-sign dimuon events in the data, while $\epsilon_{T O T}$ is measured
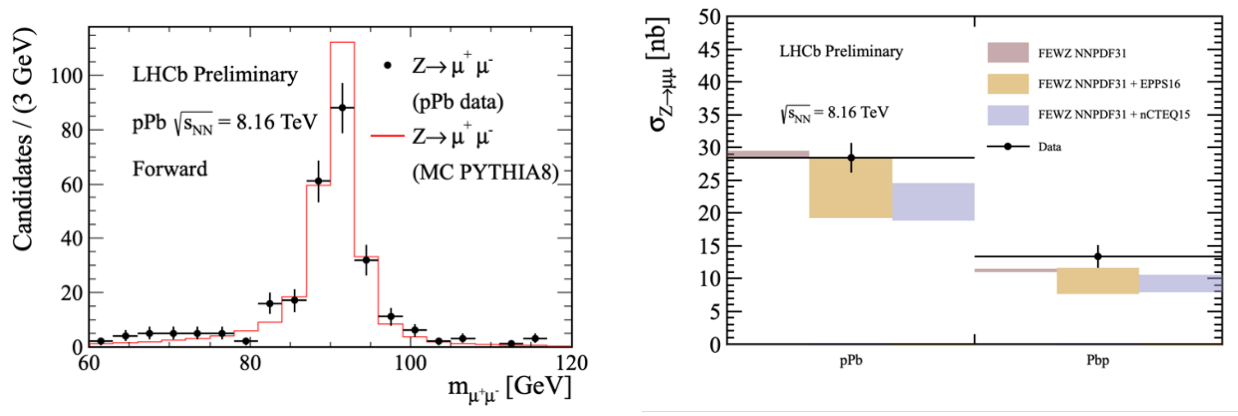

Figure 1: (Left) Dimuon invariant mass after all selection in the $p \mathrm{~Pb}$ sample, data (dots) are compared with simulation (histogram) and (right) cross-section measurement for the two configurations compared with the different nPDFs theory models discussed in the text (right).

using a combination of simulation and data-driven techniques. The results in the fiducial regions are

$$
\begin{aligned}
& \sigma_{Z \rightarrow \mu^{+} \mu^{-}}^{p P b}=28.5 \pm 1.7(\text { stat }) \pm 1.2(\text { syst }) \pm 0.7(\text { lumi }) \mathrm{nb} \\
& \sigma_{Z \rightarrow \mu^{+} \mu^{-}}^{P b p}=13.4 \pm 1.0(\text { stat }) \pm 1.4(\text { syst }) \pm 0.3(\text { lumi }) \mathrm{nb},
\end{aligned}
$$

where the first uncertainty is statistical, the second systematic and the third is due to the measurement of the luminosity. In Fig. 1 the measurements are compared to several theoretical computations using the FEWZ [11, 12] model with the NNPDF 3.1 [13] for the proton PDF and NNPDF3.1 PDFs as well as EPPS16(NLO) [8] and nCTEQ15(NLO) [7, 14] nPDFs for the lead beams. The measurement is consistent with the theory within the uncertainty, with a slight preference for the model without nPDFs effects. This result greatly improves in precision w.r.t. the previous

\footnotetext{
${ }^{1}$ The luminosity used for this analysis is $12.2 \pm 0.3$ and $18.6 \pm 0.5 \mathrm{nb}^{-1}$ for the $p \mathrm{~Pb}$ and $\mathrm{Pb} p$ sample respectively, slightly
} smaller than the total one reported in tab. 1. 
LHCb result [15] and it is consistent with the recent measurements of the same quantity by other experiments [9]. The ratio of $\sigma_{Z \rightarrow \mu^{+} \mu^{-}}$for forward and backward configurations, $R_{F B}$, is also measured, as this quantity is particularly sensitive to nuclear effects and it can be measured precisely since many systematic effects cancel in the ratio. The measurement of $R_{F B}$ in the common rapidity region $\left(2.5<\left|y^{*}\right|<4.0\right)$ in the centre-of-mass frame of the produced $\mathrm{Z}$ boson is found to be $R_{F B}\left(2.5<\left|y^{*}\right|<4.0\right)=1.28 \pm 0.14$ (stat) \pm 0.14 (syst) \pm 0.05 (lumi), compatible with theoretical calculations in the same $y^{*}$ range using FEWZ with the same nPDFs choices as for the cross section.

\section{4. $\mathrm{J} / \psi$ photo-production in ultra-peripheral $\mathrm{PbPb}$ collisions}

A collision is called ultra-peripheral when the two colliding nuclei barely miss each other, such that the impact parameter is larger than the sum of their two radii. Despite the fact that they do not physically touch each other, they can however interact by exchanging a photon, and this interaction can be greatly enhanced by the strong electromagnetic field of the nucleus. The interaction can be coherent ( $\gamma$ interacts with the nucleus as a whole) or incoherent ( $\gamma$ interacts with one or more of the nucleons in the nucleus). The measurement of the rate of coherent charmonia production can be extremely important to constrain the gluon PDFs, while the ratio of production of different charmonia states can discriminate on inputs in certain color dipole models [16].

The $\mathrm{J} / \psi$ coherent cross section has been measured as a function of rapidity in $\mathrm{PbPb}$ collisions at $\mathrm{LHCb}$ using the 2015 dataset at $\sqrt{s_{\mathrm{NN}}}=5.02 \mathrm{TeV}$, according to the formula

$$
\frac{d \sigma_{\text {coherent }}^{\mathrm{J} / /}}{d y}=\frac{N_{\text {coherent }}^{\mathrm{J} / \psi}}{\epsilon_{\text {tot }} \cdot \mathcal{L} \cdot \Delta y \cdot B\left(\mathrm{~J} / \psi \rightarrow \mu^{+} \mu^{-}\right)} .
$$

The events are selected by requiring two long tracks (the muons) and nothing else in the detector, where the new HERSCHEL detector [17] is used for this requirement. The signal candidate events are then determined by a fit on the dimuon invariant mass in the kinematic range $2.0<\eta^{\mu}<4.5$, $p_{\mathrm{T}}^{\mu}>700 \mathrm{MeV} / c, p_{\mathrm{T}}^{\mu \mu}>1 \mathrm{GeV} / c$ and $\left|\Delta \phi_{\mu \mu}\right|>0.9 \pi$, shown in Fig. 2, left. The coherent component is separated by the incoherent one through a fit on the momentum of the dimuon system shown in Fig. 2, right. The coherent $\mathrm{J} / \psi$ cross section as a function of rapidity is shown in Fig. 3, where the
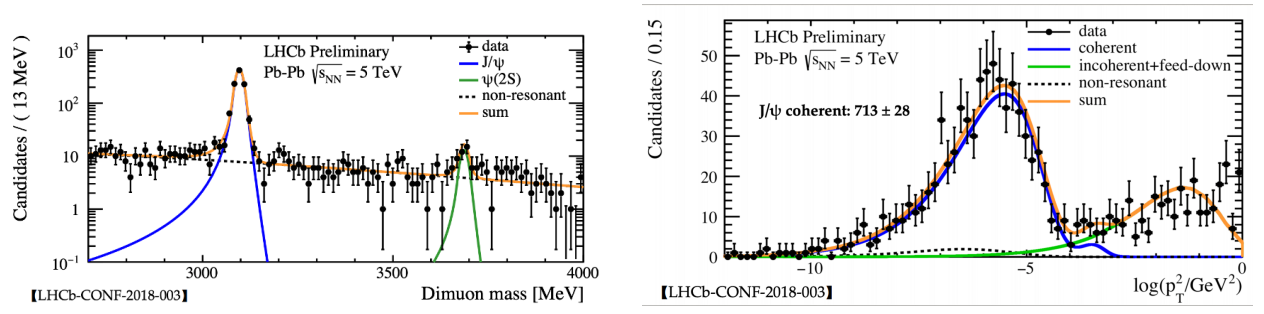

Figure 2: (Left) Dimuon invariant mass after all selection in the $\mathrm{PbPb}$ sample, the data (dots) are overlayed to the results of the fit (histogram) and (right) transverse momentum distribution of the same events. The coherent contribution is well visible in the low $p_{\mathrm{T}}$ region in the region of $\log \left(p_{\mathrm{T}}{ }^{2} / \mathrm{GeV} / c^{2}\right)$ between -10 and -4 .

results are compared to several theoretical models using perturbative QCD calculations [18] and 

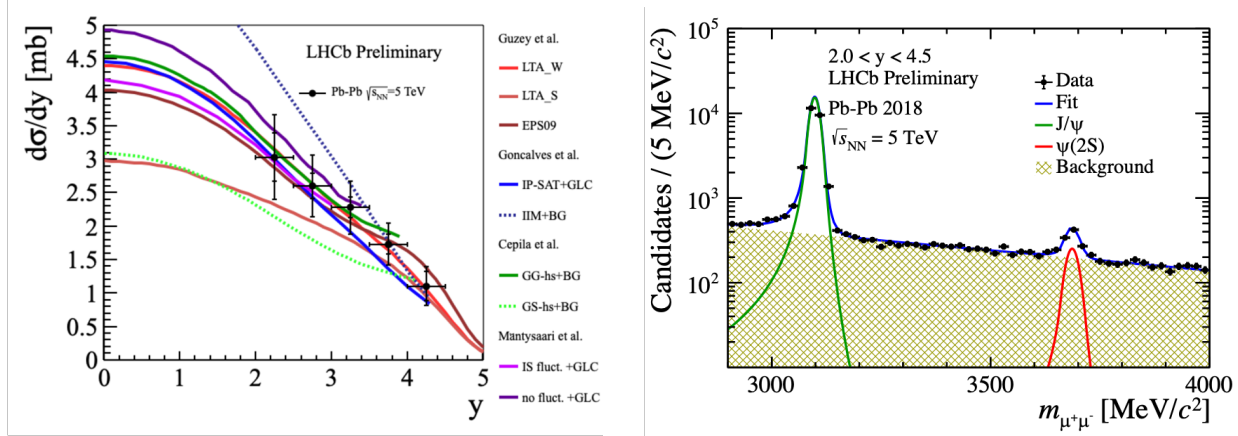

Figure 3: (Left) Differential $\mathrm{J} / \psi$ cross section measurement as a function of rapidity in UPC $\mathrm{PbPb}$ collisions. The data (dots) are compared to several theoretical prediction discussed in the text. (Right) Dimuon invariant mass in $2018 \mathrm{PbPb}$ collisions.

color dipole models $[16,19,20]$. The measurement has the potential to allow great discrimination among the different models in the region at lower rapidity where the differences between the theories are more pronounced. More precise results are expected by the analysis of the $2018 \mathrm{PbPb}$ sample, roughly 20 times larger; the dimuon invariant mass for this sample is shown in Fig. 3, where both the $\mathrm{J} / \psi$ and $\psi(2 S)$ are clearly visible [21].

\section{Conclusions and outlook}

$\mathrm{LHCb}$ is in a unique position to probe the nuclear PDFs in the region of forward rapidity with a precision unreachable by other current experiments, crucial to constrain the thoretical models. Results in $p \mathrm{~Pb}$ and $\mathrm{PbPb}$ will be even more precise in Run 3, starting in 2022, where a brand new detector is expected to reach centralities up to $30 \%$ in $\mathrm{PbPb}$ collisions, and a data size significantly larger than the Run $2 p \mathrm{~Pb}$ and $\mathrm{PbPb}$ samples, contributing to estabilishing $\mathrm{LHCb}$ as a dominant figure in Heavy Ion Physics in the near future.

\section{References}

[1] LHCв collaboration, The LHCb detector at the LHC, JINST 3 (2008) S08005.

[2] LHCв collaboration, LHCb detector performance, Int. J. Mod. Phys. A30 (2015) 1530022 LHCB-DP-2014-002, CERN-PH-EP-2014-290, [1412 . 6352].

[3] T. Pierog, I. Karpenko, J.M. Katzy, E. Yatsenko and K. Werner, EPOS LHC: Test of collective hadronization with data measured at the CERN Large Hadron Collider, Phys. Rev. C92 (2015) 034906 [1306.0121].

[4] S. Klein, J. Nystrand, J. Seger, Y. Gorbunov and J. Butterworth, Starlight: A monte carlo simulation program for ultra-peripheral collisions of relativistic ions, Computer Physics Communications 212 (2017) 258. 
[5] T. Sjöstrand, S. Mrenna and P. Skands, A brief introduction to PYTHIA 8.1, Comput. Phys. Commun. 178 (2008) 852 [0710.3820].

[6] Geant4 collaboration, Geant4 developments and applications, IEEE Trans.Nucl.Sci. 53 (2006) 270.

[7] A. Kusina, F. Lyonnet, D. Clark, E. Godat, T. Jezo, K. Kovarik et al., Vector boson production in $\mathrm{pPb}$ and $\mathrm{PbPb}$ collisions at the LHC and its impact on $n C T E Q 15$ PDFs, Eur. Phys. J. C 77 (2017) 488 [1610.02925].

[8] K.J. Eskola, P. Paakkinen, H. Paukkunen and C.A. Salgado, EPPS16: Nuclear parton distributions with LHC data, Eur. Phys. J. C 77 (2017) 163 [1612 . 05741].

[9] LНСв collaboration, Measurement of the $Z$ production cross-sections in pPb collisions at $\sqrt{=8} \mathrm{TeV}$, LHCb-CONF-2019-003 (2019) .

[10] D. Stump, J. Huston, J. Pumplin, W.-K. Tung, H. Lai, S. Kuhlmann et al., Inclusive jet production, parton distributions, and the search for new physics, JHEP 10 (2003) 046 [hep-ph/0303013].

[11] R. Gavin, Y. Li, F. Petriello and S. Quackenbush, FEWZ 2.0: A code for hadronic Z production at next-to-next-to-leading order, Comput. Phys. Commun. 182 (2011) 2388.

[12] Y. Li and F. Petriello, Combining QCD and electroweak corrections to dilepton production in FEWZ, Phys. Rev. D 86 (2012) 094034 [1208. 5967].

[13] NNPDF collaboration, Parton distributions from high-precision collider data, Eur. Phys. J. C 77 (2017) 663 [1706.00428].

[14] K. Kovarik et al., nCTEQ15 - Global analysis of nuclear parton distributions with uncertainties in the CTEQ framework, Phys. Rev. D93 (2016) 085037 [1509. 00792].

[15] LHCв collaboration, Observation of Z production in proton-lead collisions at LHCb, JHEP 09 (2014) 030 CERN-PH-EP-2014-126 LHCb-PAPER-2014-022, [1406 . 2885].

[16] H. Mäntysaari and B. Schenke, Probing subnucleon scale fluctuations in ultraperipheral heavy ion collisions, Phys. Lett. B 772 (2017) 832 [1703.09256].

[17] K. Carvalho Akiba et al., The HeRSCheL detector: high-rapidity shower counters for LHCb, JINST 13 (2018) P04017 LHCb-DP-2016-003, [1801. 04281].

[18] V. Guzey, E. Kryshen and M. Zhalov, Coherent photoproduction of vector mesons in ultraperipheral heavy ion collisions: Update for run 2 at the CERN Large Hadron Collider, Phys. Rev. C 93 (2016) 055206 [1602 . 01456].

[19] V.P. Gonçalves, M.V.T. Machado, B. Moreira, F.S. Navarra and G.S. dos Santos, Color dipole predictions for the exclusive vector meson photoproduction in $p p, p P b$, and $\mathrm{PbPb}$ collisions at run 2 LHC energies, Phys. Rev. D 96 (2017) 094027 [1710 . 10070]. 
[20] J. Cepila, J.G. Contreras and M. Krelina, Coherent and incoherent $\mathrm{J} / \psi$ photonuclear production in an energy-dependent hot-spot model, Phys. Rev. C 97 (2018) 024901.

[21] LHCв collaboration, "https://twiki.cern.ch/twiki/bin/view/LHCb/LHCbPlotsQM2019.” 